\title{
Clinical Study \\ Effects of Metformin on the Cerebral Metabolic Changes in Type 2 Diabetic Patients
}

\author{
Yung-Cheng Huang, ${ }^{1}$ Chien-Chin Hsu, ${ }^{1}$ Wei-Che Lin, ${ }^{2}$ Tang-Kai Yin, ${ }^{3}$ Chi-Wei Huang, \\ Pei-Wen Wang, ${ }^{1,5}$ Han-Hsuan Chang, ${ }^{1}$ and Nan-Tsing Chiu ${ }^{6}$ \\ ${ }^{1}$ Department of Nuclear Medicine, Kaohsiung Chang Gung Memorial Hospital and Chang Gung University College of Medicine, \\ 123 Dapi Road, Niaosong District, Kaohsiung 833, Taiwan \\ ${ }^{2}$ Department of Diagnostic Radiology, Kaohsiung Chang Gung Memorial Hospital and Chang Gung University College of Medicine, \\ 123 Dapi Road, Niaosong District, Kaohsiung 833, Taiwan \\ ${ }^{3}$ Department of Computer Science and Information Engineering, 700 Kaohsiung University Road, Nanzih District, \\ Kaohsiung 811, Taiwan \\ ${ }^{4}$ Department of Neurology, Kaohsiung Chang Gung Memorial Hospital and Chang Gung University College of Medicine, \\ 123 Dapi Road, Niaosong District, Kaohsiung 833, Taiwan \\ ${ }^{5}$ Division of Endocrinology and Metabolism, Department of Internal Medicine, Kaohsiung Chang Gung Memorial Hospital and \\ Chang Gung University College of Medicine, 123 Dapi Road, Niaosong District, Kaohsiung 833, Taiwan \\ ${ }^{6}$ Department of Nuclear Medicine, National Cheng Kung University Hospital, College of Medicine, National Cheng Kung University, \\ 138 Shengli Road, Tainan 70428, Taiwan
}

Correspondence should be addressed to Nan-Tsing Chiu; ntchiu@mail.ncku.edu.tw

Received 3 January 2014; Accepted 20 February 2014; Published 24 March 2014

Academic Editors: A. Ciarmiello and A. Varvarigou

Copyright (C) 2014 Yung-Cheng Huang et al. This is an open access article distributed under the Creative Commons Attribution License, which permits unrestricted use, distribution, and reproduction in any medium, provided the original work is properly cited.

Metformin, a widely used antidiabetic drug, has numerous effects on human metabolism. Based on emerging cellular, animal, and epidemiological studies, we hypothesized that metformin leads to cerebral metabolic changes in diabetic patients. To explore metabolism-influenced foci of brain, we used 2-deoxy-2- $\left[{ }^{18} \mathrm{~F}\right]$ fluoro-D-glucose (FDG) positron emission tomography for type 2 diabetic patients taking metformin (MET, $n=18$ ), withdrawing from metformin (wdMET, $n=13$ ), and not taking metformin (noMET, $n=9$ ). Compared with the noMET group, statistical parametric mapping showed that the MET group had clusters with significantly higher metabolism in right temporal, right frontal, and left occipital lobe white matter and lower metabolism in the left parahippocampal gyrus, left fusiform gyrus, and ventromedial prefrontal cortex. In volume of interest (VOI-) based group comparisons, the normalized FDG uptake values of both hypermetabolic and hypometabolic clusters were significantly different between groups. The VOI-based correlation analysis across the MET and wdMET groups showed a significant negative correlation between normalized FDG uptake values of hypermetabolic clusters and metformin withdrawal durations and a positive but nonsignificant correlation in the turn of hypometabolic clusters. Conclusively, metformin affects cerebral metabolism in some white matter and semantic memory related sites in patients with type 2 diabetes.

\section{Introduction}

Metformin, (N,N-dimethylimidodicarbonimidic diamide hydrochloride; commercial name: Glucophage, Glumetza, Riomet), a widely used antihyperglycemic drug administered orally for the management of type 2 diabetes, has numerous effects on human metabolism [1, 2]. It has the ability to decrease hepatic glucose production and intestinal absorption and improve insulin sensitivity by increasing peripheral glucose uptake and utilization. Additionally, it lowers blood cholesterol and triglyceride levels, reducing the risk of developing heart disease. Unlike sulfonylureas, metformin does not cause hyperinsulinemia in either type 2 diabetic patients or normal subjects, insulin secretion 
remaining unchanged. The association between metformin and dementia has recently been reported in cellular models, animal models, and epidemiological studies. Metformin can cross the blood-brain barrier and have specific pharmacological effects on the central nervous system (CNS) [3]. The exact mechanism and sites of its action in the CNS remain uncertain, however.

Metformin has recently attracted much attention because of its possibly beneficial effects on the CNS. Metformin may attenuate CNS-based inflammation, protect against apoptotic cell death in primary cortical neurons, and promote neurogenesis, and it is a potential therapy for injured or degenerating nervous system in cellular and animal models [4-7]. In contrast, one study [8] reported that metformin could deregulate $\beta$-secretase (BACE1) promoter activity and induce more than twice the normal production of $\beta$-amyloid peptide $(\mathrm{A} \beta)$, the protein that forms toxic brain plaques in Alzheimer's disease (AD).

Epidemiological evidence $[9,10]$ suggests that diabetes increases the risk of dementia; diabetes and dementia are two of the most common and devastating health problems in the elderly. "Diabetes dementia" is probably a mix of vascular and neurodegenerative dementia. Two populationbased epidemiological studies about dementia and metformin administration had contrasting results: a decreased risk of dementia observed in Taiwan [11], but a slightly increased risk in long-term users in the UK [12].

Based on these studies, we hypothesized that metformin causes cerebral metabolic changes in type 2 diabetic patients. Because a lack of human data about metformin's effects on cerebral metabolism, we used 2-deoxy-2-[ $\left.{ }^{18} \mathrm{~F}\right]$ fluoro-Dglucose (FDG) positron emission tomography (PET) to scan patients with type 2 diabetes and then analyzed the scans using statistical parametric mapping (SPM).

\section{Materials and Methods}

This study was approved by the Institutional Review Board of Chang Gung Memorial Hospital.

2.1. Patients. Adults with type 2 diabetes who were taking antidiabetic drugs were recruited prospectively. Exclusion criteria were neuropsychological or medical conditions that can alter mental status, alcohol or substance abuse, using hypnotics during the previous 2 weeks, brain tumor, autoimmune disease, AIDS, pregnancy, and a history of head trauma with a loss of consciousness. Detailed information about the study was given to all participants, and all signed written informed consent forms before they entered the study. At the visits, medical and trauma histories were reviewed, glycated hemoglobin $\left(\mathrm{HbA}_{1 \mathrm{c}}\right)$ levels were checked, and the MiniMental State Examination (MMSE) was administered and the scores recorded. Finally, 40 patients were enrolled. Based on their metformin usage, they were categorized into three groups: (i) MET: patients taking metformin ( $n=18$ ); (ii) wdMET: patients who were taking metformin but had begun withdrawing from it more than 3 days before the FDG PET scan $(n=13)$; and (iii) noMET: patients who had not taken metformin $(n=9)$. All participants, including the patients not taking metformin (noMET), were subjected to FDG PET scan for the purpose of cancer screening. This study is not considered a clinical drug trial but a clinical observational study.

2.2. FDG PET. FDG PET studies were done using a combined PET/CT scanner (Discovery ST; GE Healthcare, Waukesha, WI, USA). The blood glucose cutoff level that contraindicated FDG injection was $11.1 \mathrm{mmol} / \mathrm{L}$. Supplemental insulin was not given to lower the blood sugar just before the FDG injection because we were concerned that the insulin would alter the FDG distribution. Participants fasted for at least $6 \mathrm{~h}$ before they were injected with $370-555 \mathrm{MBq}(10-$ $15 \mathrm{mCi}$ ) of FDG. They then rested for $60 \mathrm{~min}$ in a quiet room, with the lights dimmed and their eyes closed. The participants were instructed to refrain from reading, listening to music, and talking during the uptake period. The brain scan consisted of a 1-field-of-view CT scan followed by a 15min PET study in 2D mode. Transaxial 2D PET data from the brain scan were reconstructed using an ordered subsets expectation maximization algorithm (OSEM) (2 iterations, 30 subsets) as $128 \times 128$-pixel images and a slice thickness of $3.27 \mathrm{~mm}$. The CT data were used for PET attenuation correction.

2.3. SPM Analyses. SPM5 (Wellcome Department of Imaging Neuroscience, University College of London, UK) implanted in MATLAB 7.7 (The MathWorks, Natick, MA, USA) was used to analyze images. PET images were interpolated (trilinearly) to a size of $2 \times 2 \times 2 \mathrm{~mm}$ voxels, spatially normalized to the standard PET template embedded in SPM5 and then smoothed with a Gaussian kernel (fullwidth at half-maximum $=10 \mathrm{~mm}$ ). Global normalization and proportional scaling with 0.8 threshold masking were applied. The three-way voxel-wise analyses were compared using two-sample $t$-tests in a covariance model that included age, body mass index (BMI), fasting blood sugar, and years of education as nuisance variables. $T$ contrast was used, and the results were at threshold with $P<$ 0.005 (uncorrected for multiple comparisons), a criterion used in several previous studies [13, 14], with an extent threshold of 150 voxels ( 1 voxel $=8 \mathrm{~mm}^{3}$ ) over whole brain regions. The results were displayed using the SPM5 extension xjView 8.1 (http://www.alivelearn.net/xjview8/), the peak coordinates for each significant cluster were labeled for specific anatomic location using Talairach Daemon 2.4.2 (http://www.talairach.org/), and tissue type (both white and gray matter) was verified by visual inspection. We used the SPM5 extension MarsBaR toolbox 0.43 (http://marsbar.sourceforge.net/) to extract individual adjusted, normalized FDG uptake values from the eligible clusters showing significant differences in the voxel-wise comparison between the MET and noMET groups. The regional FDG uptake values were then used to evaluate the differences between the three groups and to analyze correlations with the time intervals from the last dose of 
TABLE 1: Patient characteristics.

\begin{tabular}{lccc}
\hline & MET $(n=18)$ & wdMET $(n=13)$ & noMET $(n=9)$ \\
\hline Gender $(\mathrm{M}: \mathrm{F})$ & $8: 10$ & $9: 4$ & $6: 3$ \\
Age $($ years $)$ & $63.8 \pm 7.1$ & $58.7 \pm 8.3$ & $60.8 \pm 5.6$ \\
BMI $\left(\mathrm{kg} / \mathrm{m}^{2}\right)$ & $25.8 \pm 3.4$ & $26.5 \pm 2.4$ & $27.7 \pm 5.5$ \\
$\mathrm{HbA}_{1 \mathrm{c}}(\%)$ & $7.0 \pm 1.0$ & $7.1 \pm 1.1$ & $6.3 \pm 0.7$ \\
Fasting blood sugar $(\mathrm{mmol} / \mathrm{L})$ & $7.5 \pm 1.4$ & $7.3 \pm 1.1$ & $7.4 \pm 1.4$ \\
Metformin daily dose $(\mathrm{mg})$ & $1194 \pm 546$ & $1058 \pm 560$ & - \\
Diabetes duration (years) & $8.2 \pm 7.1$ & $7.9 \pm 8.2$ & $7.1 \pm 9.0$ \\
$\quad$ y<5:y $\geq 5$ & $7: 11$ & $8: 5$ & $5: 4$ \\
MMSE score & $25.6 \pm 4.3$ & $27.0 \pm 2.2$ & $27.0 \pm 2.9$ \\
Education (years) & $9.7 \pm 6.4$ & $9.9 \pm 6.2$ & $10.4 \pm 5.1$ \\
Current smoker & 1 & 2 & 2 \\
\hline
\end{tabular}

Values are means \pm standard deviation or numeric proportions, as indicated. MET: patients taking metformin; wdMET: patients withdrawing from metformin for more than 3 days; noMET: patients not taking metformin; BMI: body mass index; $\mathrm{HbA}_{1 \mathrm{c}}$ : glycated hemoglobin; MMSE: Mini-Mental State Examination. There were no significant differences between the three groups (all $P>0.05$ ).

metformin to the FDG injection (withdrawal durations) in the MET and wdMET groups.

2.4. Statistical Analyses. Differences in gender, age, BMI, $\mathrm{HbA}_{1 \mathrm{c}}$, fasting blood sugar, metformin daily dose, diabetes duration, MMSE score, and education between groups were compared using a $\chi^{2}$ test and one-way analysis of variance (ANOVA), as appropriate. Group differences in FDG uptake values in eligible clusters were examined using one-way ANOVA followed by two-tailed post hoc Student's $t$-test. Associations between normalized FDG uptake values in eligible clusters and metformin withdrawal durations were assessed using Pearson correlations. SPSS 20 for Windows (SPSS Inc., Chicago, IL, USA) was used for the statistical analyses. Significance was set at $P<0.05$.

\section{Results}

3.1. Patient Characteristics. All patients were right-handed. There were no significant differences in gender, age, BMI, $\mathrm{HbA}_{1 \mathrm{c}}$, fasting blood sugar, diabetes duration, MMSE score, education, or smoking between groups (Table 1). The prescribed daily dose of metformin was not significantly different between the MET and wdMET groups.

3.2. Voxel-Wise SPM between Group Comparisons. The MET group had areas with significantly $(P<0.005$, uncorrected, $k>150$ ) higher metabolism than did the noMET group in the white matter of the right temporal, right frontal, and left occipital lobes (Figure 1(a)); the wdMET group had areas with significantly $(P<0.005$, uncorrected, $k>150)$ higher metabolism in the same areas than did the noMET group, but they were smaller than those in the MET group (Figure 1(b)). In the MET group, metabolism was significantly $(P<0.005$, uncorrected, $k>150$ ) lower in the parahippocampal gyrus $(\mathrm{PH})$ of the left limbic lobe, the fusiform gyrus (FG) of the left temporal lobe (Brodmann area 37), and the ventromedial prefrontal cortex (VMPFC), including foci in the right orbital gyrus (Brodmann area 11), left rectal gyrus (Brodmann area
11), and right medial frontal gyrus (Figure 1(a)) than in the noMET group. In the wdMET group, metabolism was significantly $(P<0.005$, uncorrected, $k>150)$ lower than in the noMET group only in the $\mathrm{PH}$ of the left limbic lobe (Figure 1(b)). In the comparison between the MET and wdMET groups, SPM analysis detected subtle differences in only one white-matter focus. Compared with the wdMET group, the MET group showed an area with significantly $(P<$ 0.005 , uncorrected, $k>150$ ) higher metabolism in the white matter of the right frontal lobe (Figure 1(c)); however, there was no suprathreshold cluster of lower metabolism.

3.3. Volume of Interest (VOI-) Based Group Comparisons and Correlation Analyses. Based on the results of the SPM group comparison between the MET and noMET groups (Table 2), we used the statistically significant clusters as VOIs (Figures 2(a) and 2(b)) and extracted the FDG uptake values in those eligible clusters from the individual normalized images using the MarsBaR toolbox. There were significant differences $(P<$ 0.001) in the average FDG uptake values of white-matter clusters between the three groups. From the MET group to the wdMET group to the noMET group, there was a clear tendency toward lower FDG uptake values (Figure 2(c)). Within the VOIs, the average FDG uptake values of the MET group were significantly higher than those of the wdMET group $(P=0.049)$, and those of the wdMET group were significantly higher than those of the noMET group $(P=$ $0.003)$. There were also significant differences $(P<0.001)$ in the average FDG uptake values of the PH, FG, and VMPFC clusters between the three groups. From the MET group to the wdMET group to the noMET group, there was a clear tendency toward higher FDG uptake values (Figure 2(d)). Within the VOIs, the average FDG uptake values of the MET group were significantly lower than those of the wdMET group $(P=0.020)$, and those of the wdMET group were significantly lower than those of the noMET group $(P=$ $0.010)$.

In VOI-based correlation analysis of the imaging findings across the MET and wdMET groups, there was a significant 


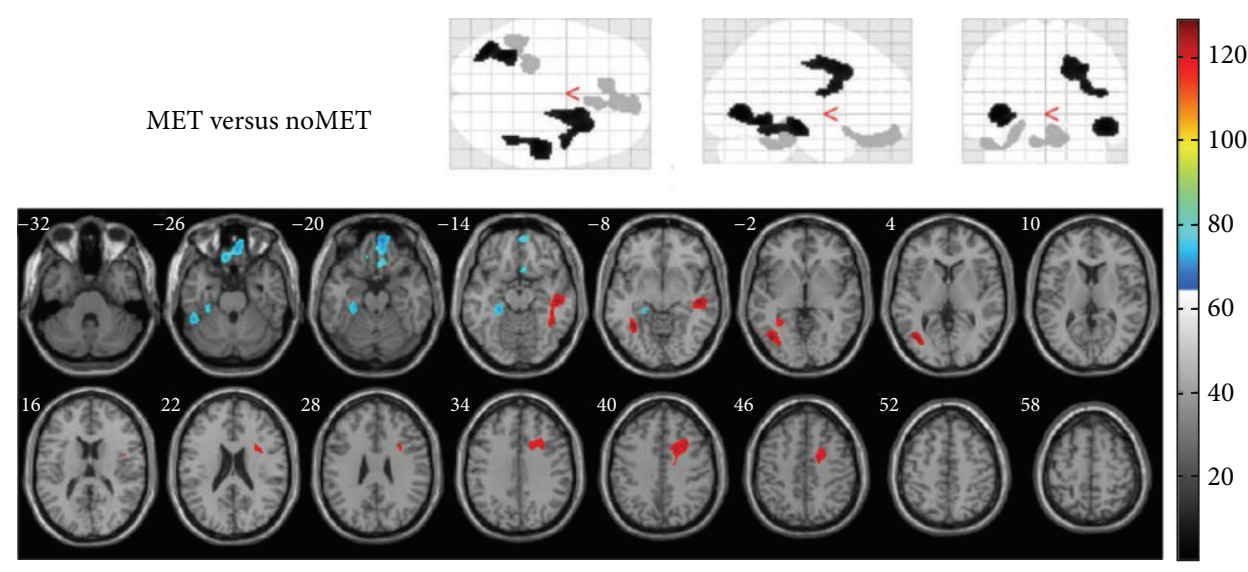

(a)

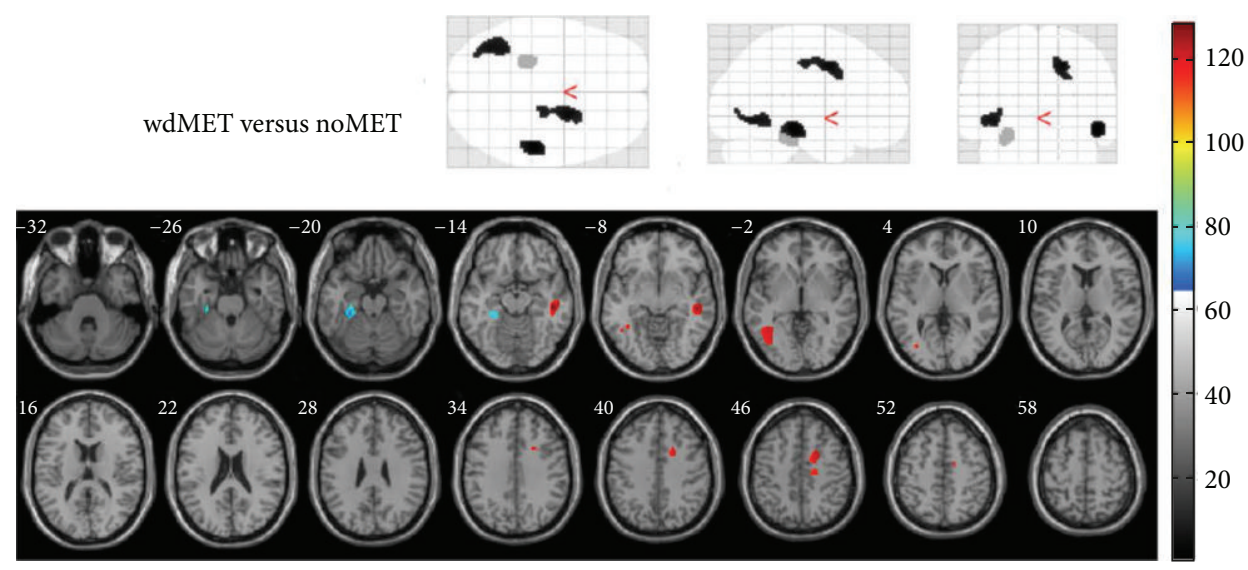

(b)

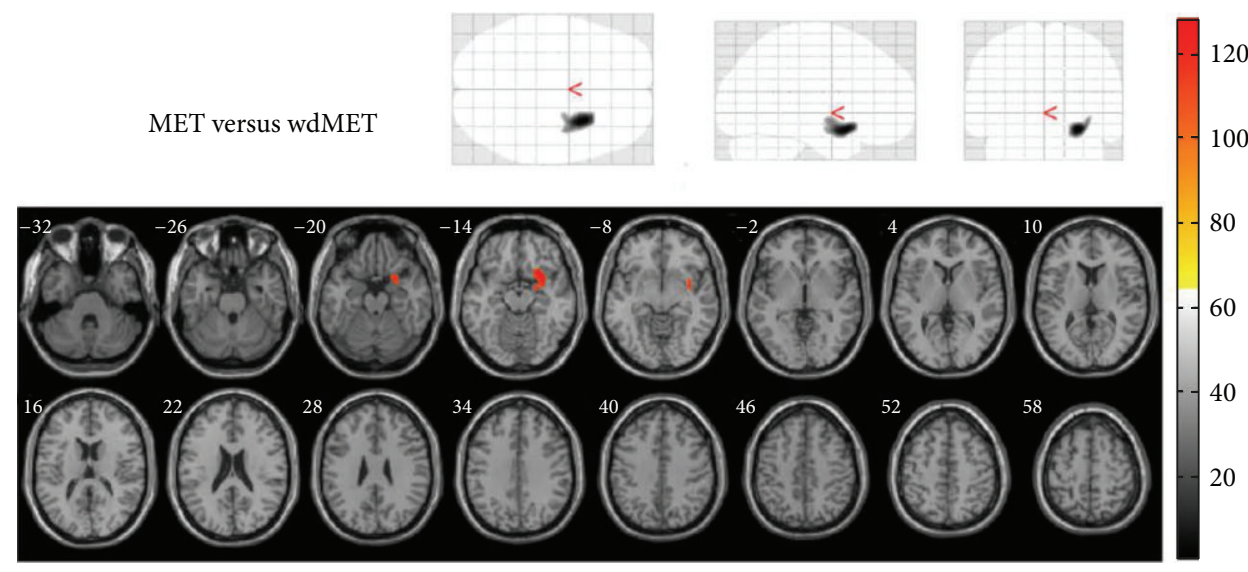

(c)

FIGURE 1: SPM analysis to compare cerebral FDG uptake between MET-group patients (taking metformin), noMET-group patients (not taking metformin), and wdMET-group patients (withdrawing from metformin). (a) MET versus noMET; (b) wdMET versus noMET; and (c) MET versus wdMET. The voxel-wise comparisons included age, body mass index, fasting blood sugar, and education as nuisance variables. Top: SPM glass brain render of the statistical voxel-wise comparisons (black: hypermetabolic regions in the former; light gray: hypometabolic regions in the former). Bottom: SPM results, slice renders of the significant clusters displayed on a T1 template overlaid with magnetic resonance images by SPM5 extension xjView 8.1 (red: hypermetabolic regions in the former; blue: hypometabolic regions in the former). All results are presented at a threshold of $P<0.005$, uncorrected, $k>150$ voxels. 
TABLE 2: SPM results of the difference in FDG metabolism between groups.

\begin{tabular}{|c|c|c|c|c|c|}
\hline \multirow{2}{*}{ Comparison groups } & \multirow{2}{*}{ Cluster size } & \multicolumn{3}{|c|}{ Voxel level } & \multirow{2}{*}{ Anatomic locations } \\
\hline & & $T$ value & $Z$ score & MNI coordinates $(x, y, z)$ & \\
\hline \multirow{7}{*}{ MET > noMET } & \multirow{2}{*}{421} & 4.58 & 3.77 & $48,-22,-10$ & \multirow{2}{*}{ Right temporal lobe, subgyral white matter } \\
\hline & & 3.38 & 2.99 & $42,-44,-14$ & \\
\hline & \multirow{2}{*}{401} & 4.70 & 3.84 & $-40,-68,0$ & Left occipital lobe, subgyral white matter \\
\hline & & 4.51 & 3.73 & $-34,-54,-6$ & Left temporal lobe, subgyral white matter \\
\hline & \multirow{3}{*}{683} & 3.87 & 3.32 & $30,14,40$ & Right frontal lobe, subgyral white matter \\
\hline & & 3.84 & 3.31 & $18,10,38$ & \multirow{2}{*}{ Right limbic lobe, white matter } \\
\hline & & 3.84 & 3.30 & $20,-2,46$ & \\
\hline \multirow{5}{*}{ MET $<$ noMET } & 217 & 4.35 & 3.63 & $-24,-32,-14$ & Left limbic lobe, parahippocampal gyrus \\
\hline & 160 & 4.20 & 3.54 & $-48,-44,-26$ & Left temporal lobe, fusiform gyrus (BA 37) \\
\hline & \multirow{3}{*}{623} & 4.94 & 3.98 & $10,56,-22$ & Right frontal lobe, orbital gyrus (BA 11) \\
\hline & & 4.00 & 3.41 & $-8,34,-26$ & Left frontal lobe, rectal gyrus (BA 11) \\
\hline & & 3.16 & 2.83 & $10,28,-20$ & Right frontal lobe, medial frontal gyrus (BA 25) \\
\hline \multirow{6}{*}{ wdMET > noMET } & 230 & 4.99 & 3.82 & $50,-26,-12$ & Right temporal lobe, subgyral white matter \\
\hline & \multirow{3}{*}{237} & 3.89 & 3.21 & $-40,-62,-4$ & \multirow{2}{*}{ Left occipital lobe, subgyral white matter } \\
\hline & & 3.05 & 3.11 & $-34,-74,-2$ & \\
\hline & & 3.73 & 3.11 & $-38,-54,-2$ & Left temporal lobe, subgyral white matter \\
\hline & \multirow{2}{*}{249} & 4.22 & 3.41 & $20,2,46$ & \multirow{2}{*}{ Right frontal lobe, subgyral white matter } \\
\hline & & 3.27 & 2.82 & $18,-18,48$ & \\
\hline wdMET < noMET & 175 & 5.26 & 3.95 & $-30,-34,-22$ & Left limbic lobe, parahippocampal gyrus \\
\hline MET > wdMET & 340 & 4.07 & 3.53 & $28,14,-14$ & Right frontal lobe, subgyral white matter \\
\hline MET < wdMET & - & - & - & - & No suprathreshold cluster \\
\hline
\end{tabular}

Threshold at $P<0.005$, uncorrected, $k>150$ voxels. MET: patients taking metformin; wdMET: patients withdrawing from metformin for more than 3 days; noMET: patients not taking metformin; BA: Brodmann area.

negative correlation $(r=-0.417, P=0.020)$ between the individual average FDG uptake values of the three white-matter clusters and metformin withdrawal durations (Figure 2(e)). In contrast, there was a positive but nonsignificant correlation $(r=0.280, P=0.127)$ between the individual average FDG uptake values of the PH, FG, and VMPFC clusters and metformin withdrawal durations (Figure 2(f)). That is, the longer a patient had been withdrawing from metformin, the more reduction of hypermetabolic changes in those affected white matter was. However, in hypometabolic PH, FG, and VMPFC, the association between withdrawal duration and metabolism restoration was not significant.

\section{Discussion}

Previous cellular and animal-model studies prompted the hypothesis that metformin is associated with change in brain metabolism. The previous animal studies [5-8], however, used metformin doses 3-10 times higher than those normally used in humans with diabetes. To explore the effects of a clinical dose of metformin on the brain metabolism in type 2 diabetic patients, we investigated functional changes of FDG PET scan using a voxel-wise analysis.

Unexpectedly, our voxel-wise analysis showed that patients in the MET group had several clusters of significantly increased metabolism in the subgyral white matter of the bilateral cerebral hemispheres, as compared with the patients in the noMET group. A possible explanation for metformin-induced hypermetabolic change of white matter is metformin-related vitamin B12 deficiency, which has been associated with the severity of cerebral white matter lesions [15]. We did not measure our patients' plasma concentrations of vitamin B12 and its related markers beforehand to support the inference of this unexpected finding. However, after we reviewed the complete blood count data (a 2-month window) available from 19 patients in the MET and wdMET groups, we found that none had an elevated mean corpuscular volume or megaloblastic anemia, which is one of the hallmarks of a metformin-induced vitamin B12 deficiency. Consequently, these white matter hypermetabolic changes are less likely caused by metformin-related vitamin B12 deficiency. This hypermetabolic change reasonably might result from metformin-induced inflammation in these white-matter clusters. FDG PET is useful for detecting infection and inflammation [16], including white matter inflammation [17] and therefore can be used to monitor neuroinflammation [18]. Metformin can more than double the production of $\mathrm{A} \beta$ [8]. $\mathrm{A} \beta$ deposits have been detected in the cerebral white matter of the $\mathrm{AD}$ brain, and their distribution corresponded to the orientation of the blood vessels [19]. Activated microglial cells were found colocalized with perivascular deposits of $\mathrm{A} \beta$ in the $\mathrm{AD}$ brain and seemed to be involved in clearing these deposits [20]. Moreover, hypermetabolism of white matter may be associated with glial cells [21]. High FDG uptake, caused by inflammation as well as microglial 


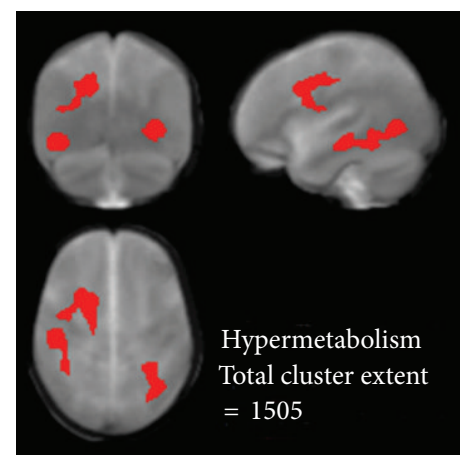

(a)

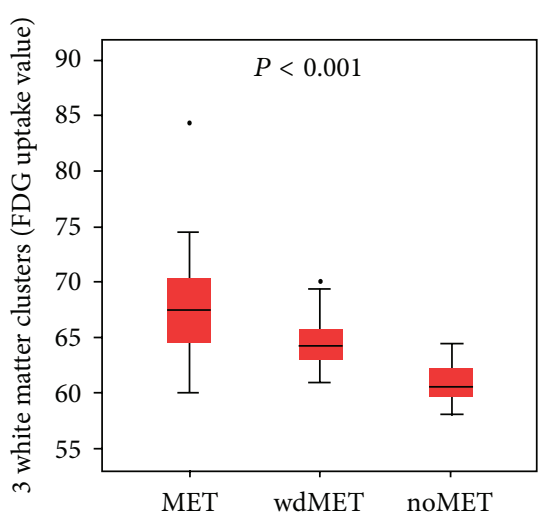

(c)

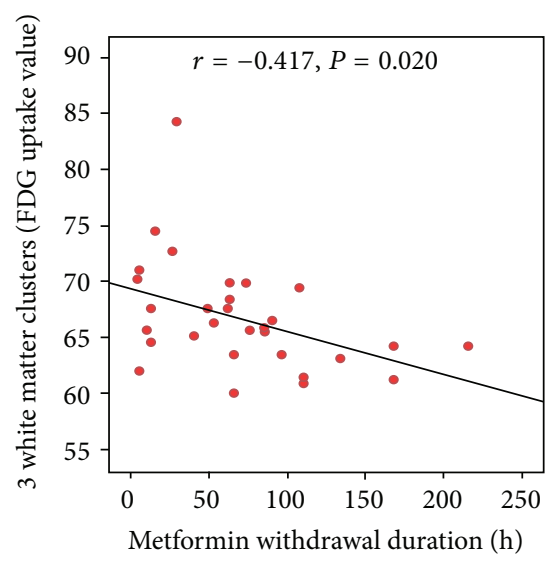

(e)

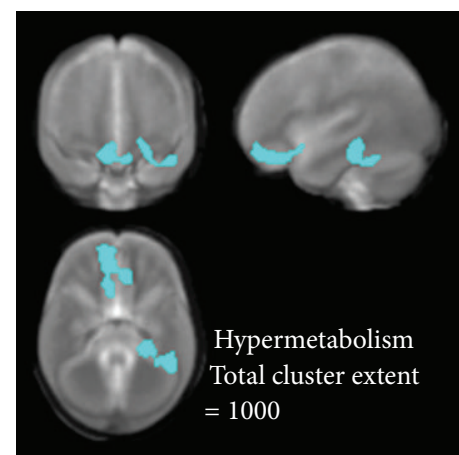

(b)

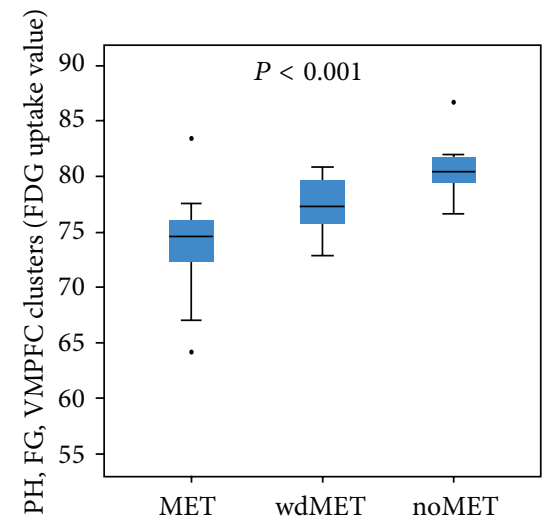

(d)

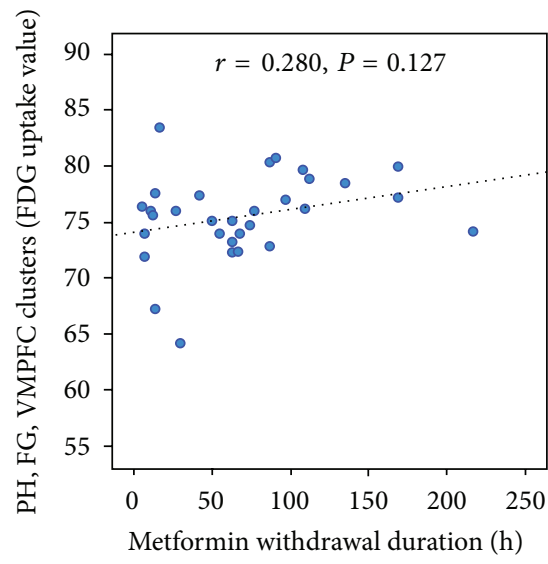

(f)

Figure 2: VOI-based (Top) group comparisons (middle) and correlation analyses (bottom). Top: (a) the red VOIs overlaid on volume rendering magnetic resonance images represent hypermetabolic clusters obtained from the voxel-wise comparison $($ MET $>$ noMET, $P<$ 0.005 , uncorrected, $k>150$ voxels). (b) The blue VOIs overlaid on volume rendering magnetic resonance images represent hypometabolic clusters obtained from the voxel-wise comparison (MET $<$ noMET, $P<0.005$, uncorrected, $k>150$ voxels). Middle: VOI-based group comparisons between the MET $(n=18)$, wdMET $(n=13)$, and noMET $(n=9)$ groups. Box plots (median, interquartile range, and extreme values) for normalized FDG uptake values in hypermetabolic clusters (c) and in hypometabolic clusters (d) between the 3 groups $(P<0.001)$. Bottom:VOI-based correlation analyses across the MET and wdMET groups. The normalized FDG uptake values in the hypermetabolic clusters (e) and in the hypometabolic clusters (f) of the patients plotted against their metformin withdrawal durations. The solid line is a significant linear regression line $(r=-0.417, P=0.020)$ and the dotted line is an estimated linear regression line $(r=0.280, P=0.127)$. FG: fusiform gyrus; MET: patients taking metformin; noMET: patients not taking metformin; $\mathrm{PH}$ : parahippocampal gyrus; VMPFC: ventromedial prefrontal cortex; VOI: volume of interest; wdMET: patients withdrawing from metformin for more than 3 days. 
cell activation, was detected in immunohistochemical data and indirectly supported by a group of patients with amyotrophic lateral sclerosis with local activated microglia $[22,23]$. Therefore, we are of the opinion that $A \beta$-associated inflammation combined with microglial cell activation causes this hypermetabolic change.

In agreement with our hypothesis, the voxel-wise group analysis found significant hypometabolic regions in the brain memory system caused by a clinical dose of metformin. Compared with the noMET group, the MET group had clusters of significantly decreased metabolism in the PH, FG, and VMPFC, which are part of the semantic memory system [24]. The VMPFC has been linked to motivation, reward processing, learning, and decision making. Lesions of the VMPFC itself affect memory monitoring and induce spontaneous confabulations [25]. Data from monkeys suggest principal connections between the VMPFC (medial orbitofrontal cortex, Brodmann area 11) and the PH areas [26], the other main foci of hypometabolism in the MET group. Patients with such lesions of the posterior medial temporal area were unable to store new information [27]. Characteristically consistent with the results of a meta-analysis [24] of functional neuroimaging studies focusing on semantic processing, the hypometabolic foci in our study were left-hemisphere lateralized. The relatively hypometabolic semantic foci in our study indicated hypofunction of those areas. This finding seems in line with the theory of the potentially harmful consequences of metformin $[8,12]$ and is supported by a clinical trial [28] which showed that metformin for monotherapy was associated with a declining trend in memory performance.

In the present study, we found that FDG uptake in metformin-influenced foci had significant trends between groups. The regional FDG uptake values used for the association showed a continuous distribution with a broad overlap between the three groups rather than group-centered clusters. This indicates that the association may not have been driven only by group-based differences. Supporting this impression, a significant negative correlation between FDG uptake values of hypermetabolic clusters in white matter and metformin withdrawal durations was also present in the MET and wdMET groups. This suggests that the influence of metformin in white matter is time-dependent on withdrawal duration, that is, on how long it has been since the patient stopped taking metformin. In contrast, there was a weak tendency toward a positive correlation between the FDG uptake values of hypometabolic clusters in semantic foci and metformin withdrawal durations, which indicates that the influence is not time-dependent on the withdrawal duration of metformin or a temporary withdrawal does not reach significant change of these long-lasting effects.

In addition to metformin, two antidiabetic agents, insulin and rosiglitazone, had been investigated for their effect on the link between diabetes and $\mathrm{AD}[8,28-30]$. In neuronal models, combination usage of insulin reduced the increased intracellular $\mathrm{A} \beta$ level caused by using metformin alone [8]. Clinical evidence [30] indicates that intranasal insulin improved cognition in patients with early AD. In clinical trials [28, 29], rosiglitazone ameliorated disease-related pathology and improved memory deficits in animal models of AD; thus, it might protect against cognitive decline in older patients with diabetes. Only one patient in the MET group used insulin and only one patient in the MET group used rosiglitazone as antidiabetic agents. After removing the data of these two patients, we did an additional SPM analysis to verify the first; the results (as shown in the Supplementary Material available online at http://dx.doi.org/10.1155/2014/694326) were similar and consistent with our formal results in Figure 1.

Because this was a pilot imaging study on the effects of metformin on brain metabolism, we were still unable to arrive at exact pharmacological mechanisms that explain some of our findings. One limitation of our study is that we did not measure circulating amyloid levels or apolipoprotein E4 as a dementia-risk allele. Additional studies with more patients and comprehensive examinations such as radionuclide imaging of amyloid, using radiolabeled PK11195 for microglial activation-associated neuroinflammation imaging, and using magnetic resonance imaging for detecting fine white matter abnormalities are needed to arrive at more powerful conclusions. In addition, we focused on metformin's effects in diabetic patients and all the enrolled patients had type 2 diabetes; therefore, we had no data on how metformin affects healthy people. We did not enroll healthy people as a control group because of ethical concerns in administration of metformin to a healthy group. Besides, cerebral glucose metabolism may be influenced to some degree in patients with diabetes. Aiming to explore the effects of metformin in type 2 diabetic patients, it is more reasonable to make comparisons between diabetic patients taking and not taking metformin.

\section{Conclusions}

The clinical dose of metformin in type 2 diabetic patients is associated with hypermetabolic changes in the white matter of the bilateral cerebral hemispheres and with hypometabolic changes in the semantic memory system (the PH, FG, and VMPFC), which is predominantly lateralized in the left hemisphere. Correlation analyses suggested that withdrawal from metformin reduces its effects on white matter hypermetabolic changes, which are duration-dependent. However, restoring hypometabolism in the PH, FG, and VMPFC was not significantly correlated with metformin withdrawal duration.

\section{Conflict of Interests}

The authors declare that there is no conflict of interests regarding the publication of this paper.

\section{Acknowledgment}

This research was supported by a grant from the Taiwan National Science Council (NSC 99-2314-B-182A-081).

\section{References}

[1] D. Kirpichnikov, S. I. McFarlane, and J. R. Sowers, "Metformin: an update," Annals of Internal Medicine, vol. 137, no. 1, pp. 25-33, 2002. 
[2] A. J. Krentz and C. J. Bailey, "Oral antidiabetic agents: current role in type 2 diabetes mellitus," Drugs, vol. 65, no. 3, pp. 385411, 2005.

[3] K. Łabuzek, D. Suchy, B. Gabryel, A. Bielecka, S. Liber, and B. Okopień, "Quantification of metformin by the HPLC method in brain regions, cerebrospinal fluid and plasma of rats treated with lipopolysaccharide," Pharmacological Reports, vol. 62, no. 5, pp. 956-965, 2010.

[4] M. El-Mir, D. Detaille, G. R-Villanueva et al., "Neuroprotective role of antidiabetic drug metformin against apoptotic cell death in primary cortical neurons," Journal of Molecular Neuroscience, vol. 34, no. 1, pp. 77-87, 2008.

[5] J. Wang, D. Gallagher, L. M. DeVito et al., "Metformin activates an atypical PKC-CBP pathway to promote neurogenesis and enhance spatial memory formation," Cell Stem Cell, vol. 11, pp. 23-35, 2012.

[6] T. C. Ma, J. L. Buescher, B. Oatis et al., "Metformin therapy in a transgenic mouse model of Huntington's disease," Neuroscience Letters, vol. 411, no. 2, pp. 98-103, 2007.

[7] N. Nath, M. Khan, M. K. Paintlia, I. Singh, M. N. Hoda, and S. Giri, "Metformin attenuated the autoimmune disease of the central nervous system in animal models of multiple sclerosis," Journal of Immunology, vol. 182, pp. 8005-8014, 2009.

[8] Y. Chen, K. Zhou, R. Wang et al., "Antidiabetic drug metformin (GlucophageR) increases biogenesis of Alzheimer's amyloid peptides via up-regulating BACE1 transcription," Proceedings of the National Academy of Sciences of the United States of America, vol. 106, no. 10, pp. 3907-3912, 2009.

[9] G. J. Biessels, S. Staekenborg, E. Brunner, C. Brayne, and P. Scheltens, "Risk of dementia in diabetes mellitus: a systematic review," The Lancet Neurology, vol. 5, no. 1, pp. 64-74, 2006.

[10] A. Ott, R. P. Stolk, F. van Harskamp, H. A. P. Pols, A. Hofman, and M. M. B. Breteler, "Diabetes mellitus and the risk of dementia: the Rotterdam study," Neurology, vol. 53, no. 9, pp. 1937-1942, 1999.

[11] C. Hsu, M. L. Wahlqvist, M. Lee, and H. Tsai, "Incidence of dementia is increased in type 2 diabetes and reduced by the use of sulfonylureas and metformin," Journal of Alzheimer's Disease, vol. 24, no. 3, pp. 485-493, 2011.

[12] P. Imfeld, M. Bodmer, S. S. Jick, and C. R. Meier, "Metformin, other antidiabetic drugs, and risk of Alzheimer's disease: a population-based case-control study," Journal of the American Geriatrics Society, vol. 60, pp. 916-921, 2012.

[13] K. Kantarci, M. L. Senjem, V. J. Lowe et al., "Effects of age on the glucose metabolic changes in mild cognitive impairment," American Journal of Neuroradiology, vol. 31, no. 7, pp. 1247-1253, 2010.

[14] B. Pascual, E. Prieto, J. Arbizu et al., "Decreased carbon-11flumazenil binding in early Alzheimer's disease," Brain, vol. 135, pp. 2817-2825, 2012.

[15] L. M. L. de Lau, A. D. Smith, H. Refsum, C. Johnston, and M. M. B. Breteler, "Plasma vitamin B12 status and cerebral white-matter lesions," Journal of Neurology, Neurosurgery and Psychiatry, vol. 80, no. 2, pp. 149-157, 2009.

[16] C. Love, M. B. Tomas, G. G. Tronco, and C. J. Palestro, "FDG PET of infection and inflammation," Radiographics, vol. 25, no. 5, pp. 1357-1368, 2005.

[17] A. E. Ramage, P. T. Fox, R. L. Brey et al., "Neuroimaging evidence of white matter inflammation in newly diagnosed systemic lupus erythematosus," Arthritis and Rheumatism, vol. 63, no. 10, pp. 3048-3057, 2011.
[18] C. G. Radu, C. J. Shu, S. M. Shelly, M. E. Phelps, and O. N. Witte, "Positron emission tomography with computed tomography imaging of neuroinflammation in experimental autoimmune encephalomyelitis," Proceedings of the National Academy of Sciences of the United States of America, vol. 104, no. 6, pp. 19371942, 2007.

[19] N. Iwamoto, E. Nishiyama, J. Ohwada, and H. Arai, "Distribution of amyloid deposits in the cerebral white matter of the Alzheimer's disease brain: relationship to blood vessels," Acta Neuropathologica, vol. 93, no. 4, pp. 334-340, 1997.

[20] T. Uchihara, H. Akiyama, H. Kondo, and K. Ikeda, "Activated microglial cells are colocalized with perivascular deposits of amyloid- $\beta$ protein in Alzheimer's disease brain,' Stroke, vol. 28, no. 10, pp. 1948-1950, 1997.

[21] H. Kita, K. Shima, M. Tatsumi, and H. Chigasaki, "Cerebral blood flow and glucose metabolism of the ischemic rim in spontaneously hypertensive stroke-prone rats with occlusion of the middle cerebral artery," Journal of Cerebral Blood Flow and Metabolism, vol. 15, no. 2, pp. 235-241, 1995.

[22] D. Fukumoto, T. Hosoya, S. Nishiyama et al., "Multiparametric assessment of acute and subacute ischemic neuronal damage: a small animal positron emission tomography study with rat photochemically induced thrombosis model," Synapse, vol. 65, no. 3, pp. 207-214, 2011.

[23] A. Cistaro, M. C. Valentini, A. Chiò et al., "Brain hypermetabolism in amyotrophic lateral sclerosis: a FDG PET study in ALS of spinal and bulbar onset," European Journal of Nuclear Medicine and Molecular Imaging, vol. 39, no. 2, pp. 251-259, 2012.

[24] J. R. Binder, R. H. Desai, W. W. Graves, and L. L. Conant, "Where is the semantic system? A critical review and meta-analysis of 120 functional neuroimaging studies," Cerebral Cortex, vol. 19, no. 12, pp. 2767-2796, 2009.

[25] A. Gilboa, "Autobiographical and episodic memory-one and the same? Evidence from prefrontal activation in neuroimaging studies," Neuropsychologia, vol. 42, no. 10, pp. 1336-1349, 2004.

[26] C. Cavada, T. Compañy, J. Tejedor, R. J. Cruz-Rizzolo, and F. Reinoso-Suárez, "The anatomical connections of the macaque monkey orbitofrontal cortex. A review," Cerebral Cortex, vol. 10, no. 3, pp. 220-242, 2000.

[27] A. Schnider, V. Treyer, and A. Buck, "Selection of currently relevant memories by the human posterior medial orbitofrontal cortex," Journal of Neuroscience, vol. 20, no. 15, pp. 5880-5884, 2000.

[28] A. M. Abbatecola, F. Lattanzio, A. M. Molinari et al., "Rosiglitazone and cognitive stability in older individuals with type 2 diabetes and mild cognitive impairment," Diabetes Care, vol. 33, no. 8, pp. 1706-1711, 2010.

[29] G. Landreth, Q. Jiang, S. Mandrekar, and M. Heneka, "PPAR $\gamma$ agonists as therapeutics for the treatment of Alzheimer's disease," Neurotherapeutics, vol. 5, no. 3, pp. 481-489, 2008.

[30] M. A. Reger, G. S. Watson, P. S. Green et al., "Intranasal insulin improves cognition and modulates $\beta$-amyloid in early $\mathrm{AD}$," Neurology, vol. 70, no. 6, pp. 440-448, 2008. 


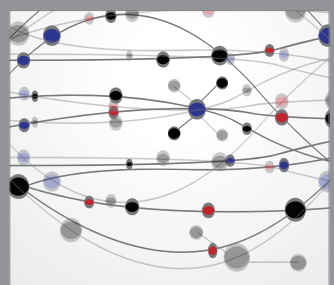

The Scientific World Journal
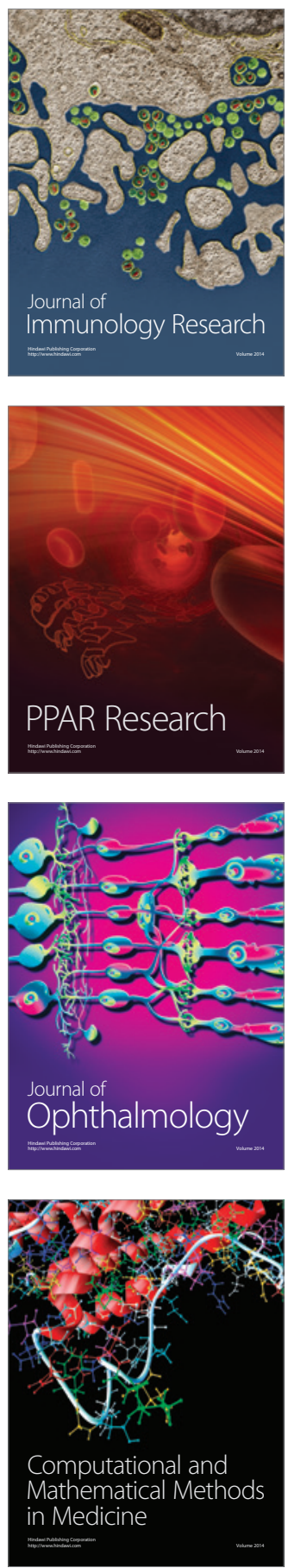

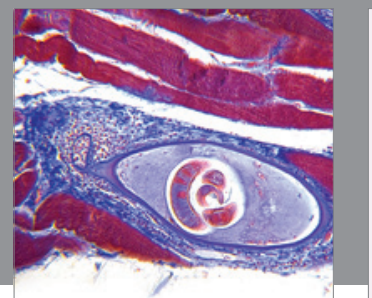

Gastroenterology

Research and Practice
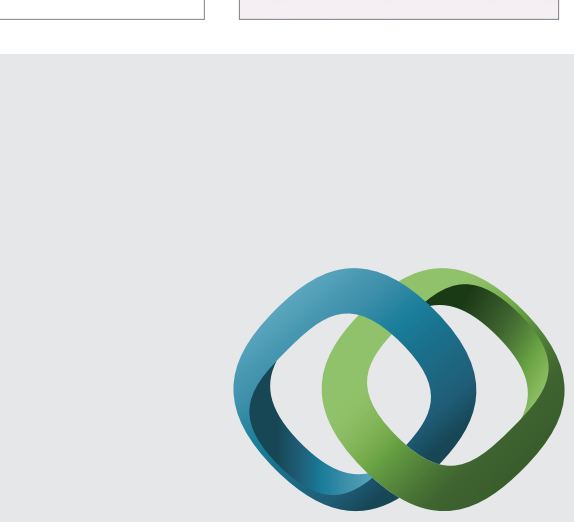

\section{Hindawi}

Submit your manuscripts at

http://www.hindawi.com
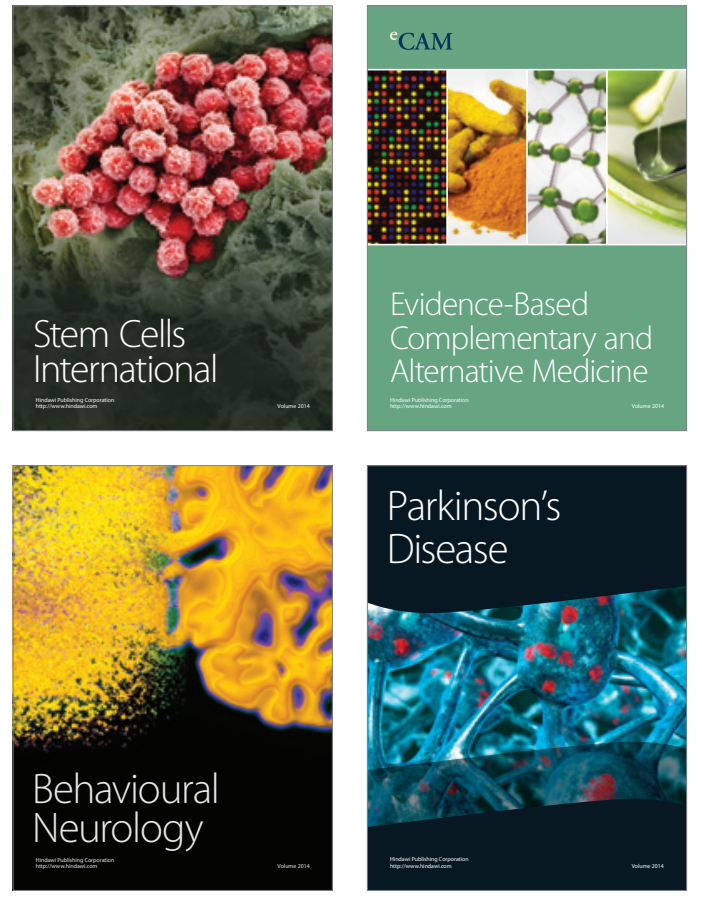
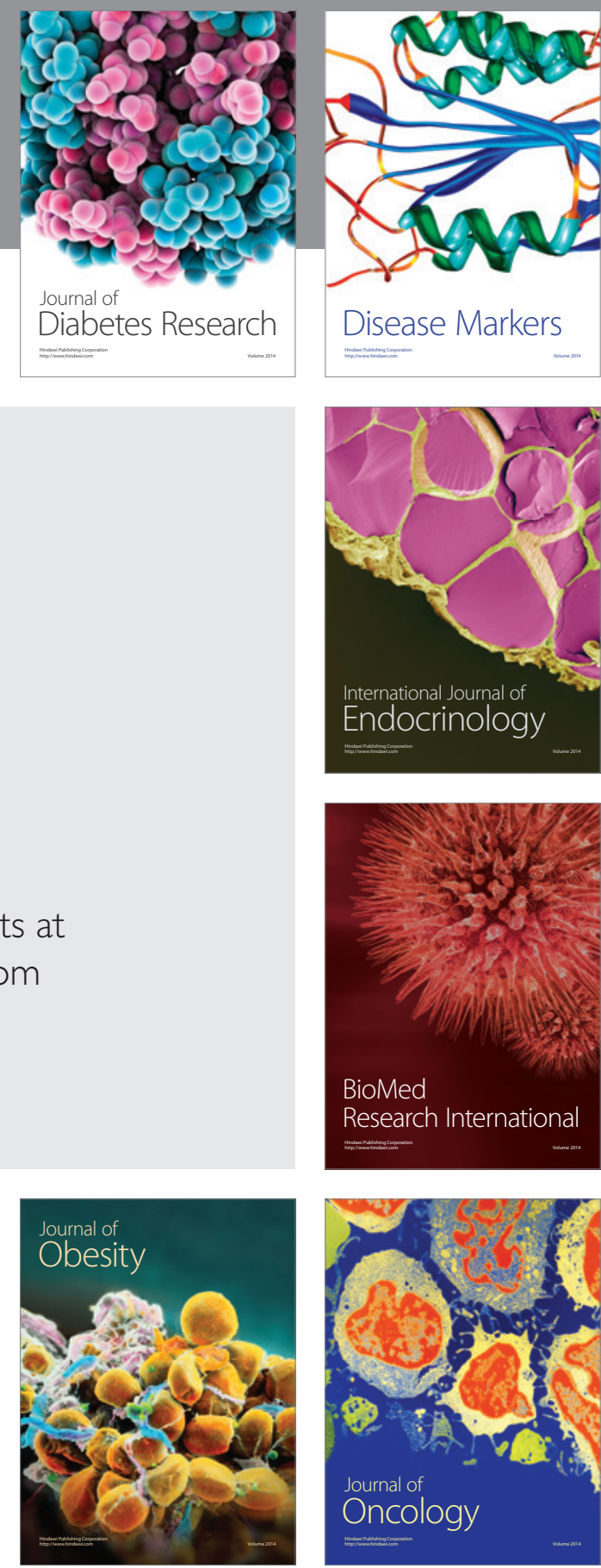

Disease Markers
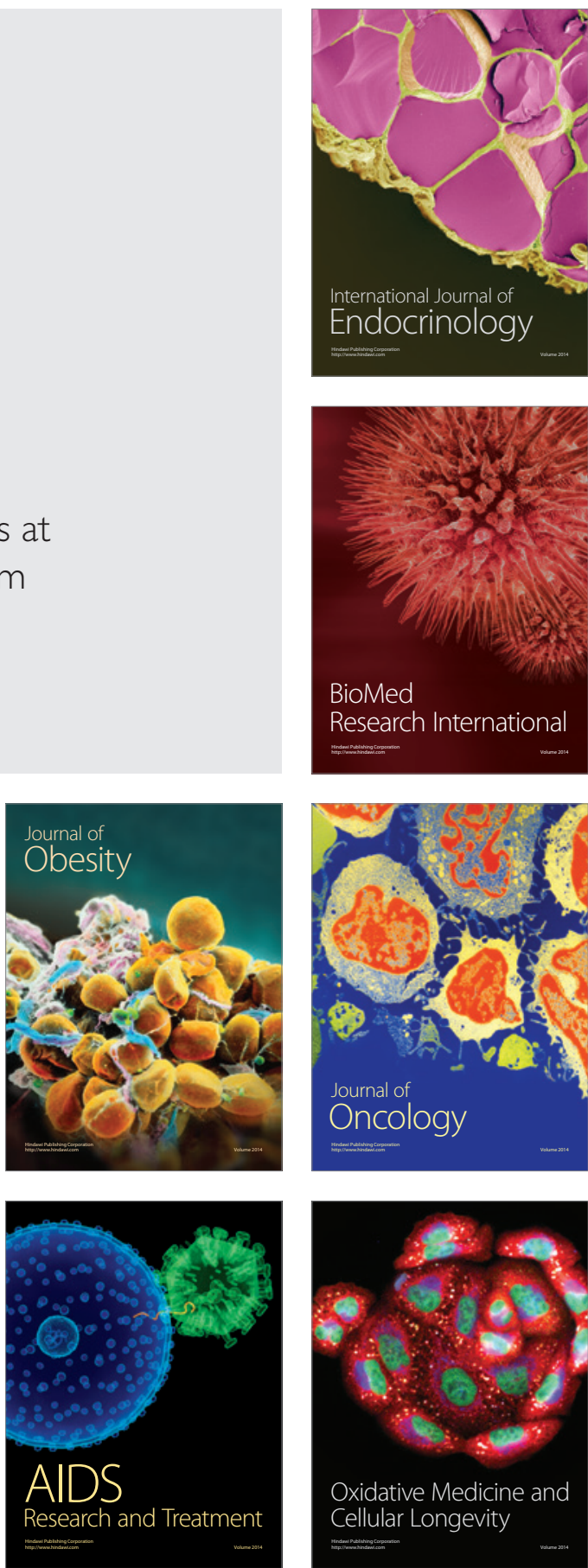